\title{
The UGT1 locus is a determinant of prostate cancer recurrence after prostatectomy
}

\author{
Isabelle Laverdière', Christine Flageole', Étienne Audet-Walsh', Patrick Caron', \\ Yves Fradet $^{2}$, Louis Lacombe ${ }^{2}$, Éric Lévesque ${ }^{1,2}$ and Chantal Guillemette ${ }^{1,3}$ \\ ${ }^{1}$ Pharmacogenomics Laboratory, Centre Hospitalier Universitaire de Québec (CHU de Québec) Research Center and \\ Faculty of Pharmacy, Laval University, R4720, 2705 Boulevard Laurier, Québec, Québec, Canada G1V 4G2 \\ ${ }^{2} \mathrm{CHU}$ de Québec Research Center and Faculty of Medicine, Laval University, Québec, Québec, Canada \\ ${ }^{3}$ Canada Research Chair in Pharmacogenomics, Québec, Québec, Canada
}

Correspondence should be addressed to C Guillemette Email chantal.guillemette@ crchudequebec.ulaval.ca

\begin{abstract}
The prognostic significance of common deletions in uridine diphospho-glucuronosyltransferase 2B (UGT2B) genes encoding sex steroid metabolic enzymes has been recently recognized in localized prostate cancer ( $\mathrm{PCa}$ ) after radical prostatectomy (RP). However, the role of germline variations at the UGT1 locus, encoding half of all human UGTs and primarily involved in estrogen metabolism, remains unexplored. We investigated whether variants of UGT1 are potential prognostic markers. We studied 526 Caucasian men who underwent RP for clinically localized PCa. Genotypes of patients for 34 haplotype-tagged single-nucleotide polymorphisms (htSNPs) and 11 additional SNPs across the UGT1 locus previously reported to mark common variants including functional polymorphisms were determined. The risk of biochemical recurrence (BCR) was estimated using adjusted Cox proportional hazards regression and Kaplan-Meier analysis. We further investigated whether variants are associated with plasma hormone levels by mass spectrometry. In multivariable models, seven htSNPs were found to be significantly associated with BCR. A greater risk was revealed for four UGT1 intronic variants with hazard ratios (HRs) of 1.59-1.88 $(P<0.002)$ for htSNPs in UGT1A10, UGT1A9, and UGT1A6. Conversely, decreased BCR was associated with three htSNPs in introns of UGT1A10 and UGT1A9 (HR=0.56-058; $P \leq 0.01)$. An unfavorable UGT1 haplotype comprising all risk alleles, with a frequency of $14 \%$, had a HR of 1.68 $(95 \% \mathrm{Cl}=1.13-2.50 ; P=0.011)$. Significant alteration in circulating androsterone levels was associated with this haplotype, consistent with changes in hormonal exposure. This study provides the first evidence, to our knowledge, that germline polymorphisms of UGT1 are potential predictors of recurrence of PCa after prostatectomy.
\end{abstract}

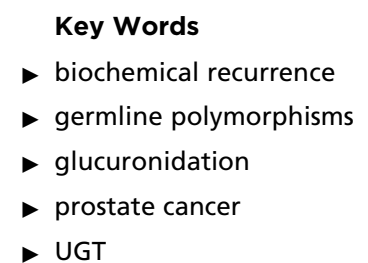

Endocrine-Related Cancer (2015) 22, 77-85

\section{Introduction}

Sex steroids play a central role in the development and progression of prostate cancer (PCa) (Mohler et al. 2012, Ryan et al. 2013). Recent evidence supports the notion that patient's genetic background influences the outcome (Audet-Walsh et al. 2011, 2012, Nadeau et al. 2011, Boyd et al. 2012, Reese et al. 2012, Levesque et al. 2013, 2014a,b, Wyatt et al. 2013). In particular, common deletions of the uridine diphospho-glucuronosyltransferase (UGT) genes UGT2B17 and UGT2B28 involved in the catabolism of androgenic sex steroids have been recognized as 
prognostic markers for localized PCa after radical prostatectomy (RP) (Nadeau et al. 2011). The UGT-mediated metabolic pathways, governed by the two gene families UGT1 and UGT2, are among key regulators of sex steroid exposure responsible for their inactivation via glucuronic acid conjugation, which prevents binding to their respective nuclear receptors and facilitates their excretion. In support of the idea that inherited genetic variations can alter the hormonal environment to which cancer cells are exposed, common gene deletions of UGT2B17 and UGT2B28 have also been associated with altered levels of circulating androgen steroids (Nadeau et al. 2011).

Half of the human UGTs are encoded by the UGT1 gene located on chromosome 2q37, which encodes nine functional enzymes. In contrast to UGT2B enzymes, UGT1As are mainly regulators of tissue exposure to estrogens, because most of them are involved in the glucuronidation of parental estrogen (estradiol $\left(\mathrm{E}_{2}\right)$ and estrone $\left(\mathrm{E}_{1}\right)$ ), and inactivation of their hydroxyl moieties at position 2 or 4 as well as methoxy catechol estrogens (Gall et al. 1999, Lepine et al. 2004, Murai et al. 2006, Starlard-Davenport et al. 2007, Zhou et al. 2010, Sneitz et al. 2013). Conversely, there is little evidence to support them having a role in the inactivation of androgens, e.g., the potent androgen, dihydrotestosterone (DHT; Gall et al. 1999, Zhou et al. 2010). UGT1A1, UGT1A3, UGT1A8, UGT1A9, and UGT1A10 conjugate $\mathrm{E}_{1}$ and $\mathrm{E}_{2}$ most efficiently, whereas UGT1A4 is less efficient. (Lepine et al. 2004, Starlard-Davenport et al. 2007, Sneitz et al. 2013). Several groups have reported that estrogens and their metabolites contribute to both the development and progression of PCa (Cavalieri \& Rogan 2011, Nelles et al. 2011, Hu et al. 2012). Genetic polymorphisms in factors that govern estrogen biosynthetic and metabolic pathways have been linked to altered exposure to sex steroid hormones and risk of PCa (Gu et al. 2014, Kanda et al. 2015). It has been reported that low-activity promoter alleles of the $\mathrm{E}_{2}$-conjugating enzyme UGT1A1 (rs3064744; $(\mathrm{TA})_{\mathrm{n}}$ repeat) are associated with a risk of low-grade PCa and a slight elevation of estrogen levels but have no gene-dosage effect (Tang et al. 2011).

Germline variations in genes involved in the biosynthesis and metabolism of androgens and estrogens have been associated with progression of PCa (Audet-Walsh et al. 2011, 2012, Nadeau et al. 2011, Levesque et al. 2013, $2014 a, b)$. The relevance of studying steroidogenic pathways in the context of progression of $\mathrm{PCa}$ arises from observations that PCa tumor cells can use multiple biotransforming pathways to de novo synthesize more potent hormones (Montgomery et al. 2008, Mitsiades et al. 2012). In support of these results, we have recently reported that singlenucleotide polymorphisms (SNPs) in genes associated with $\mathrm{E}_{2}$-related metabolic pathways, either individually or in combination, are predictors of progression of PCa. These variants are located in genes encoding cytochrome P450 $1 \mathrm{~B} 1$, which is involved in the production of 4-hydroxy metabolites of $\mathrm{E}_{2}$ and $\mathrm{E}_{1}(4-\mathrm{OH})$, catechol-O-methyltransferase, which forms 2-methoxy catechol estrogens, sulfotransferase $2 \mathrm{~B} 1$, which catalyzes the formation of less-reactive sulfate metabolites, and 17 $\beta$-hydroxysteroid dehydrogenase type 2 , which is involved in the conversion of $\mathrm{E}_{2}$ to $\mathrm{E}_{1}$ (Levesque et al. 2014a). However, it remains unclear whether the outcome of PCa is influenced by common germline polymorphisms in the estrogen catabolic pathway mediated by UGT1 gene products.

The objective of this study was to determine whether common variations across the UGT1 locus, which encodes multiple estrogen-metabolizing UGT1A enzymes, are associated with a modified risk of biochemical recurrence (BCR) after prostatectomy in patients with localized PCa and to ascertain their relationships with levels of endogenous circulating hormones. After adjustments for known clinico-pathological variables, seven UGT1 SNPs were found to be significantly associated with time to $\mathrm{BCR}$, three SNPs were protective and four were associated with an increased risk. Additional analyses identified an unfavorable UGT1 haplotype, characterized by the latter four risk markers, that is also linked with changes in exposure to circulating hormones.

\section{Materials and methods}

\section{Clinical data and outcome collections}

The cohort included 526 Caucasian patients with localized PCa. All patients underwent RP at l'Hôtel-Dieu de Québec Hospital (QC, Canada) between February 1999 and December 2002 (Audet-Walsh et al. 2011, Nadeau et al. 2011). All participants provided written informed consent for genetic analysis, and the Local Research Ethics Committee of the CHU de Québec approved the research protocol. The clinical characteristics of the studied cohort are given in Table 1 .

\section{Genetic analysis and steroid measures}

Polymorphisms of the UGT1 locus were assessed by PCR amplification by Sequenom iPLEX matrix-assisted laser desorption/ionization time-of-flight mass spectrometry. With the objective of covering most of the haplotype

Published by Bioscientifica Ltd. 
Table 1 Clinical and pathological characteristics of prostate cancer patients.

\begin{tabular}{|c|c|}
\hline Characteristics & $n(\%)$ \\
\hline Localized PCa & $526(100)$ \\
\hline Mean age at diagnosis (years) & 63.3 \\
\hline S.D. age at diagnosis (years) & 6.8 \\
\hline Range age at diagnosis & $43.5-80.7$ \\
\hline Median follow-up (months) & 88.8 \\
\hline Biochemical recurrence risk & $130(24.7)$ \\
\hline \multicolumn{2}{|l|}{ PSA at diagnosis $(\mathrm{ng} / \mathrm{ml})$} \\
\hline$\leq 10$ & $362(69)$ \\
\hline$>10-20$ & $103(20)$ \\
\hline$>20$ & $56(11)$ \\
\hline \multicolumn{2}{|l|}{ Pathological Gleason score } \\
\hline$\leq 6$ & $158(31)$ \\
\hline 7 & $244(48)$ \\
\hline$\geq 8$ & $107(21)$ \\
\hline \multicolumn{2}{|l|}{ Pathological T stage } \\
\hline $\mathrm{pT}=\mathrm{T} 2$ & $313(60)$ \\
\hline $\mathrm{pT}=\mathrm{T} 3 \mathrm{a}$ & $131(25)$ \\
\hline $\mathrm{pT} \geq \mathrm{T} 3 \mathrm{~b}$ & 77 (15) \\
\hline \multicolumn{2}{|l|}{ Nodal invasion } \\
\hline NO & $481(92)$ \\
\hline $\mathrm{N}+$ & $44(8)$ \\
\hline \multicolumn{2}{|l|}{ Neoadjuvant hormone therapy } \\
\hline Yes & $31(6)$ \\
\hline No & $495(94)$ \\
\hline \multicolumn{2}{|l|}{ Adjuvant hormone therapy } \\
\hline Yes & $30(6)$ \\
\hline No & $496(94)$ \\
\hline \multicolumn{2}{|l|}{ Margin status } \\
\hline Negative & $368(70)$ \\
\hline Positive & $154(30)$ \\
\hline \multicolumn{2}{|l|}{ Smoking status } \\
\hline Non/ex-smoker & $438(83)$ \\
\hline Smoker & $85(16)$ \\
\hline \multicolumn{2}{|l|}{ D'Amico risk classification } \\
\hline Low & 187 (36) \\
\hline Intermediate & $208(40)$ \\
\hline High & $122(24)$ \\
\hline
\end{tabular}

PCa, prostate cancer; PSA, prostate-specific antigen; T, tumor; N, node.

diversity, polymorphisms in UGT1 were chosen on the basis of results from our previous study of Caucasian volunteers recruited in the same geographical region (Menard et al. 2009) and as described by the genetics of the CEU (Utah residents with Northern and Western European ancestry) HapMap population. A region covering all exons, introns, and $5 \mathrm{~kb}$ upstream and downstream of each gene was screened using a haplotype-tagging SNP (htSNP) strategy (Audet-Walsh et al. 2011, 2012). Overall, we genotyped $34 \mathrm{htSNPs}$ (Fig. 1) with an additional 11 associated SNPs across the UGT1A locus previously reported to mark common variants in regulatory domains, exons, and intron-exon boundaries, including some functional polymorphisms (Supplementary Table 1, see section on supplementary data given at the end of this article). This included genotyping of the UGT1A1 promoter variant (rs3064744; c.-54/-53insTA) genotyped by direct sequencing of PCR-amplified products, as described previously (Girard et al. 2008). Negative controls were included for every run of analysis, and quality controls consisting of random samples were successfully performed on 5\% of the study cohort. SNPs that had 5\% missing genotypes were excluded from the analysis. None of the studied SNPs deviated from the Hardy-Weinberg equilibrium. A total of 495 plasma samples were available and collected the morning of the surgical procedure. Levels of circulating sex steroids for this cohort of patients have been reported (Nadeau et al. 2011). Data are expressed as the mean \pm the s.E.M. To ensure the validity of each analysis, quality controls that included known steroid concentrations with deuterated isotopes were included in each run of analysis.

\section{Statistical analysis}

To analyze associations between polymorphisms and BCR, each htSNP was first categorized according to homozygosity/heterozygosity (i.e. major allele homozygote, heterozygote, and minor allele homozygote), because the functions of most of these htSNPs are unknown. Rare homozygotes (frequency $<2 \%$ ) were combined with heterozygotes. Cox regression analysis was performed for each SNP with adjustment for clinico-pathological variables. Multivariable models included prostate-specific antigen (PSA) at diagnosis, Gleason score, pathological $\mathrm{T}$ stage, age at diagnosis, surgical margins, nodal invasion status, smoking status, neoadjuvant therapy, adjuvant therapy. All covariables were treated as categorical, and $\leq 4 \%$ of all covariables had missing values. The censoring variable was BCR, as reported previously (Nadeau et al. 2011). Haplotypes were inferred using Phase version 2.1.1 (Stephens et al. 2001, Stephens \& Donnelly 2003). Pairwise linkage disequilibrium was determined with HAPLOVIEW 3.32 (www.broad.mit.edu/mpg/haploview). Kaplan-Meier analyses (log-rank) and univariate Cox regression analyses were performed for each SNP. As we tested multiple polymorphisms $(n=45)$, false-discovery rates ( $q$ values) were calculated to determine the degree to which the tests were prone to false positives using the R QVALUE package (http://genomics.princeton.edu/storeylab/qvalue/).

Analysis of covariance was used to compare means of each natural log-transformed hormonal variable between haplotype groups adjusted for age. Patients with missing genotype or hormone level data were excluded. For comparisons among haplotype groups, any significant

Published by Bioscientifica Ltd 


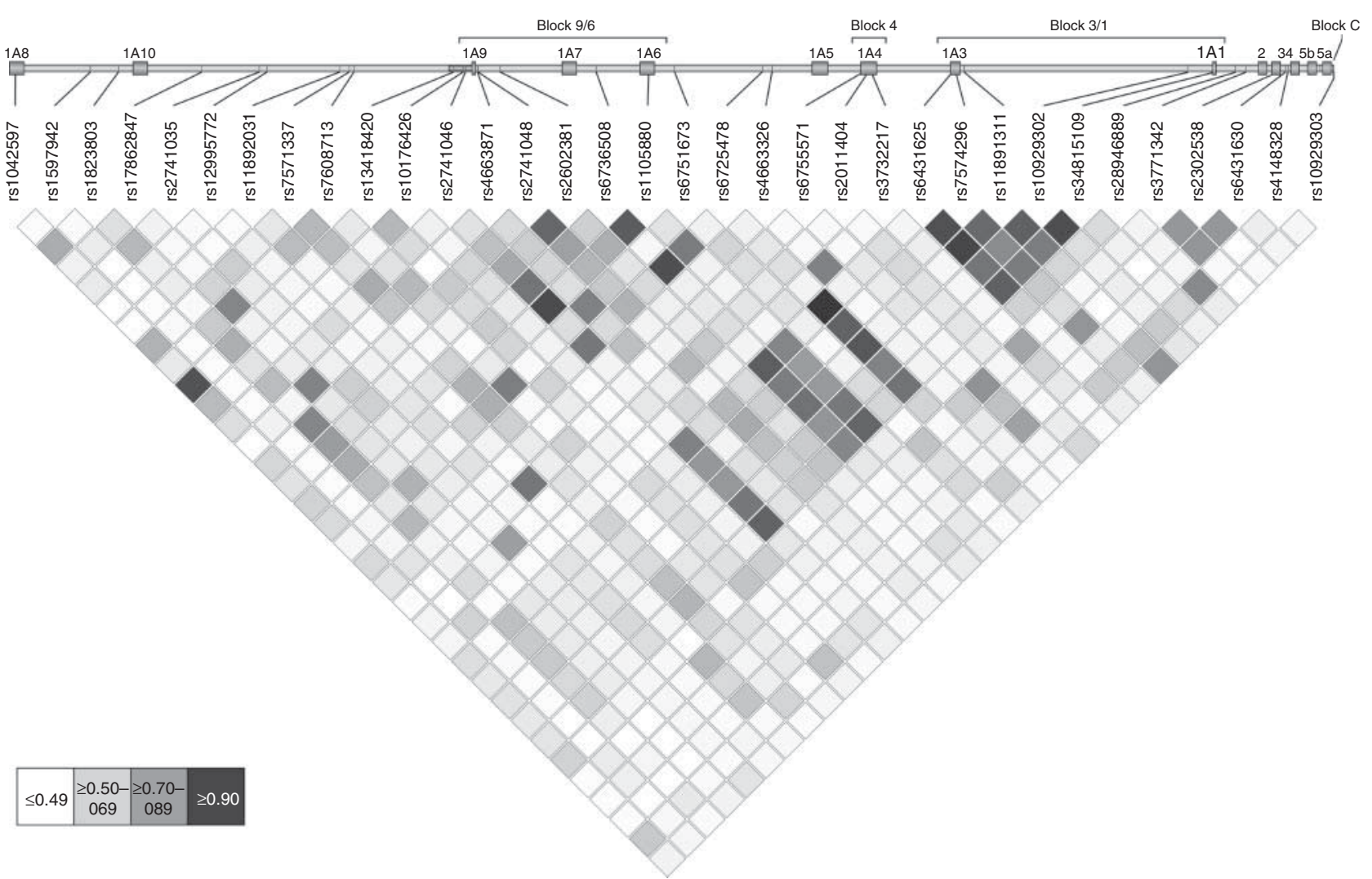

Figure 1

UGT1 common germline variations genotyped in the cohort of 526 Caucasian men with clinically localized prostate cancer and treated by radical prostatectomy. We genotyped 34 htSNPs and 11 additional associated SNPs across the UGT1 locus previously reported to mark

differences revealed by analysis of covariance were further investigated using Fisher's least significant difference post-hoc pairwise comparison. Patients who had previously received androgen deprivation therapy were excluded from our analyses. Statistical analyses were performed using PASW Statistics version 17 (SPSS, Inc.).

\section{Results}

Overall, $24.7 \%$ of patients experienced recurrence after prostatectomy (median follow-up time, 7.4 years). None of the germline variations tested was associated with the main predictors of BCR (PSA at diagnosis, Gleason score, pathological $\mathrm{T}$ stage, and surgical margin status; Supplementary Table 2 , see section on supplementary data given at the end of this article). After adjustments for known prognostic factors had been made, seven variants located in the intronic or regulatory regions of UGT1 were associated with an altered risk of BCR. The relative frequency of each htSNP and its corresponding hazard common variants in regulatory domains, exons, and intron-exon boundaries, including some functional polymorphisms. Pairwise linkage disequilibrium ( $r^{2}$ values) is graphically represented by different shades of gray.

ratio (HR; 95\% CI) are presented in Table 2 . One variation in the UGT1A10 (rs1823803) and two variations in the UGT1A9 intronic region (rs2741048 and rs2602381) were associated with a significantly decreased risk of BCR with HR values between 0.56 and $0.58(P<0.01)$. Conversely, htSNPs located in the UGT1A10 intronic region (rs17862847 and rs7608713) and in the UGT1A9 intronic region (rs4663871) were associated with an increased risk for $\mathrm{BCR}(\mathrm{HR}=1.59-1.88 ; P<0.02)$. In addition, one variation rs6751673 in UGT1A6 was positively associated with BCR and displayed an increased risk of events $(\mathrm{HR}=1.68 ; P=0.008)$. The Kaplan-Meier curves showing the BCR-free survival for the UGT1 markers significantly associated with BCR and their logrank $P$ values are available in the supplementary material (Supplementary Fig. 1, see section on supplementary data given at the end of this article). No significant association was observed for any of the known functional UGT1 variants evaluated, e.g., the TATA box promoter variant UGT1A1*28 (rs34815109; c.-54/-53insTA), UGT1A3 V47A

Published by Bioscientifica Ltd 


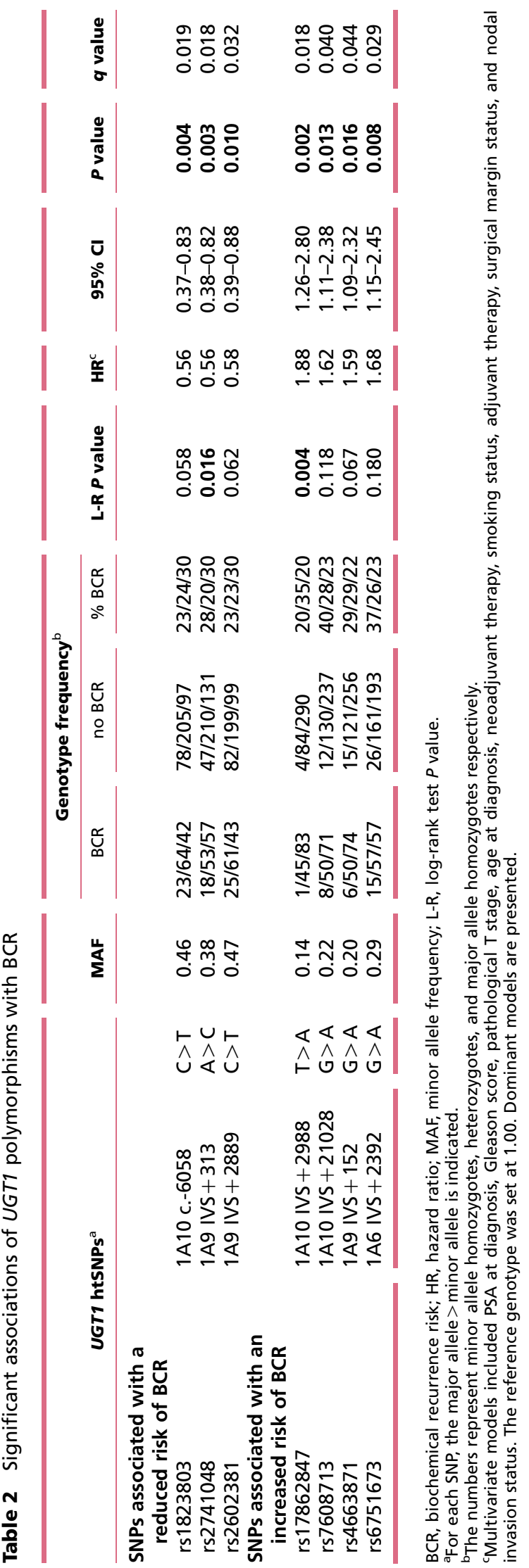

(rs6431625; c.140T >C), or other non-synonymous common variations located in exons UGT1A3 and UGT1A4, and in the common exons $2-5$ or the $3^{\prime}$ UTR.

Five haplotypes inferred using the seven positive UGT1 markers are significantly associated with the occurrence of BCR at an allelic frequency of $\geq 5 \%$ in our cohort. The occurrence rates of BCR were $23,22,33,21$, and 19\%, respectively, for haplotypes 1-5 (H1-H5; Fig. 2A). After adjustments for other variables, we found that H3, characterized by the presence of the four unfavorable alleles and no protective allele, displayed a greater risk of BCR (Table 3). H3 carriers display a HR of $1.68(P=0.011)$ compared with the reference $\mathrm{H} 1$ that includes none of the increased risk and/or the protective alleles described earlier. We also observed that individuals who carry both $\mathrm{H} 3$ and two or more deleted copies of UGT2B17 and UGT2B28 tend to have decreased BCR-free survival as shown by the Kaplan-Meier curves for BCR. The BCR-free stratifications are 71 and 55\% respectively, when $\mathrm{H} 3$ is sub-classified according to the number of UGT2B deletions ( $0-1$ UGT2B deletion $(n=112)$ versus $\geq 2$ $U G T 2 B$ deletions ( $n=33)$; Fig. 2B).

We also compared the mean levels of plasma unconjugated sex steroids and androgen glucuronides for H3 carriers with only zero or one UGT2B deletion, because individuals with more than two UGT2B deletions had been previously reported to present reduced circulating levels of DHT metabolites, namely androsterone glucuronide (ADT-G) and $3 \alpha$-androstanediol-17G (Nadeau et al. 2011). Compared with non-carriers of UGT1 risk alleles (i.e. H1), carriers of $\mathrm{H} 3$ presented a $15 \%$ lower level of ADT $(206.30 \pm 9.83 \mathrm{pg} / \mathrm{ml}(n=112)$ versus $176.21 \pm 11.93 \mathrm{pg} / \mathrm{ml}(n=50) ; P=0.01)$, whereas no significant changes in androgen glucuronides, $\mathrm{E}_{2}$, and $\mathrm{E}_{1}$ could be detected (data not shown).

\section{Discussion}

We provide evidence for the prognostic value of UGT1 for localized PCa. As has been observed UGT2B deletions, we found that several UGT1 htSNPs are associated with an increased risk of recurrence. The markers positively linked with a greater risk of BCR are located in intronic regions of exons 1A6, 1A9, and 1A10. This finding supports our hypothesis that genetic variants of UGT1 may modify the progression of $\mathrm{PCa}$ - probably by altering sex steroid metabolism - although direct biological evidence for this hypothesis is lacking.

We had previously reported a significant decrease in circulating androgen metabolites, namely ADT-G and

Published by Bioscientifica Ltd. 

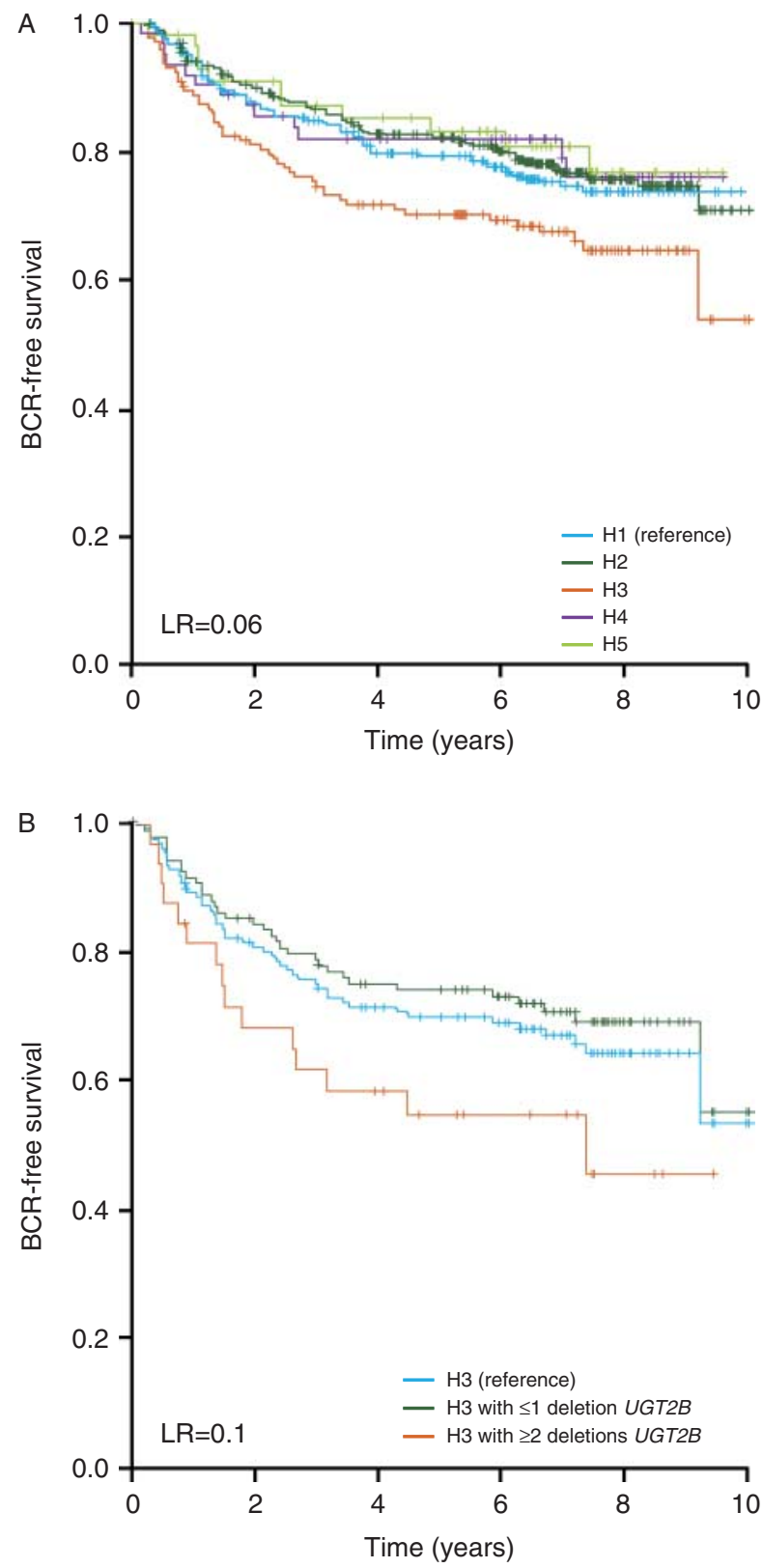

Figure 2

Kaplan-Meier curves for (A) the five most frequent UGT1A haplotypes associated with BCR in the cohort of Caucasian men with localized PCa $(n=526)$ and (B) the high-risk haplotype 3 combined with UGT2B17 and UGT2B28 deletions. Log-rank (LR) $P$ values are reported in each frame.

$3 \alpha$-diol-17G, in PCa patients with two or more deletions in the androgen-conjugating UGT2B17 and UGT2B28 (Nadeau et al. 2011). On the basis of the substratespecificity of the nine functional UGT1As involved in estrogen catabolism, we expected to find little if any influence on formation of androgen glucuronides associated with htSNPs. In fact, few reported results support the ability of recombinant UGT1As to conjugate androgens, with the exception of UGT1A3 and UGT1A4, which have weak activity toward DHT and its metabolite ADT compared with UGT2B enzymes (Gall et al. 1999, Zhou et al. 2010). This is consistent with our observation that no significant changes in circulating androgen glucuronides are observed for carriers of UGT1-related risk haplotypes. UGT1A enzymes are well known to be involved in the metabolism of estrogens, including the parental estrogens $\mathrm{E}_{2}$ and $\mathrm{E}_{1}$, and catechol estrogens and their methoxy derivatives; however, no significant changes in circulating levels of unconjugated $\mathrm{E}_{1}$ and $\mathrm{E}_{2}$ could be detected in our study. It remains to be ascertained if levels of estrogen metabolites, including their glucuronide derivatives, are modified by variants of UGT1. We found positive markers mostly in regions related to UGT1A9 and UGT1A10, with their gene products being mainly involved in the metabolism of 2-OH- $\mathrm{E}_{2}, 4-\mathrm{OH}-\mathrm{E}_{2}$, and 2-methoxy- $\mathrm{E}_{2}$ (Lepine et al. 2004). We are currently developing a mass spectrometry assay to address whether the identified UGT markers could be involved in recurrence of PCa by promoting unbalanced glucuronidating metabolism for these specific estrogen metabolites or by their relative abundance compared with that of androgens, thereby favoring progression of cancer. The carcinogenicity of catechol oxidative metabolites has been recognized for various cancers, including PCa (Bosland \& Mahmoud 2011, Cavalieri \& Rogan 2011). Results of a pilot study by Kosti et al. (2011) indicated that some catechol estrogens are present in greater concentrations in the urine of $\mathrm{PCa}$ patients compared with that of healthy men, indicating an association between estrogen metabolites and risk of PCa. However, based on a method developed by Xu et al. (2007), they did not directly measure the levels of estrogen glucuronide derivatives but reported total estrogen concentrations (glucuronidates + sulfates + unconjugated sex steroids following hydrolysis with sulfatase and $\beta$-glucuronidase).

Very few studies have documented the expression of UGT1 gene products in human prostate. Court et al. (2012) detected nine UGT1A transcripts in a pool of human prostates and detected all UGT1As but at significantly lower levels compared with those found in liver samples. During normal prostate differentiation, downregulation of UGT1A transcripts was observed in normal prostatic stem cells compared with more differentiated cells (Williamson et al. 2013). Furthermore, assessment of individual UGT1A transcripts revealed that the expression of UGT1A8 was elevated in progenitor cells, with no change in amounts of UGT2B expressed. UGT1A8 is the major UGT1A involved in inactivation of the carcinogenic metabolites

Published by Bioscientifica Ltd. 


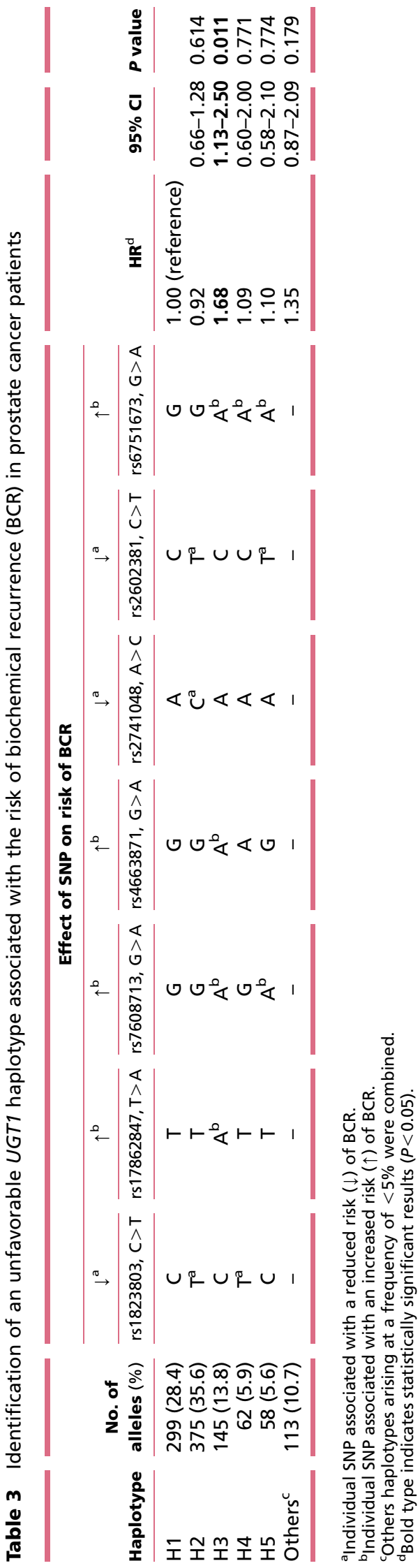

http://erc.endocrinology-journals.org DOI: $10.1530 /$ ERC-14-0423
(C) 2015 Society for Endocrinology Printed in Great Britain
4-OH-E $\mathrm{E}_{1} / \mathrm{E}_{2}$ (Lepine et al. 2004). In support of a role for UGT1As in progression of $\mathrm{PCa}$, transient repression of UGT1A expression in the PCa cell line LNCaP has been shown to contribute to activation of androgen receptors leading to upregulation of PSA along with increased cell survival in the standard steroid-containing medium (Williamson et al. 2013). This study also provided additional evidence that reduced expression of UGT1A is a feature of clinical PCa associated with a poorer prognosis and may be explained by the fact that upon depletion of UGT1A, a higher level of steroids is expected. However, Williamson and colleagues did not address whether levels of expression of UGT1A affect levels of steroids and whether estrogens rather that androgens are altered upon partial repression of UGT1A. In fact, their results obtained in vitro with LNCaP cells might be caused by the action of androgens and estrogens because both types of steroids are present in a complete medium and because androgens are converted to estrogens by the action of aromatase. A role for estrogens in this context is thus plausible because UGT1A more efficiently inactivates estrogens compared with androgens such as DHT, and it is known that estrogens are proficient in activating the mutated androgen receptor in the LNCaP cell line (Tan et al. 1997). In addition, expression of aromatase is upregulated by approximately 30-fold $(P<0.001)$ in metastatic PCa tumors compared with primary tumors (Montgomery et al. 2008), indicating a greater production of estrogens during progression of PCa. Changes in UGT1A expression have also been reported in clinical specimens. In castration-resistant patients, low levels of expression of UGT1A have been found compared with that observed in early PCa (Varambally et al. 2005, Williamson et al. 2013), as well as decreased UGT1A immunostaining in aggressive disease (Williamson et al. 2013). Altogether, these findings provide evidence supporting the suggestion that reduced expression of UGT1A is linked to poor prognosis and may represent a mechanism for accumulation of sex steroids, probably estrogens, within PCa cells. In this study linking several genetic markers of the UGT1 gene to BCR, the decreased levels of the DHT metabolite ADT in carriers of the UGT1risk haplotype may reflect enhanced production of estrogens from androgens or a modification of androgen metabolism, although additional investigations are required to validate this hypothesis.

In conclusion, our study provides the first evidence, to our knowledge, that germline polymorphisms in the UGT1 locus modify the likelihood of BCR after prostatectomy. Our findings also highlight the need to better

Published by Bioscientifica Ltd 
characterize the levels of $\mathrm{E}_{2}$ and estrogen metabolites, the main known steroid hormone substrates of UGT1A enzymes, in PCa patients. Further studies are needed to define the underlying mechanisms through which UGT1As contribute to the biology of PCa and patient outcomes.

\section{Supplementary data}

This is linked to the online version of the paper at http://dx.doi.org/10.1530/ ERC-14-0423.

\section{Declaration of interest}

The authors declare that there is no conflict of interest that could be perceived as prejudicing the impartiality of the research reported.

\section{Funding}

The Cancer Research Society (C Guillemette) and Fonds de Recherche en Santé du Québec (FRQ-S) supported this work. I Laverdière, C Flageole, and É Audet-Walsh were recipients of Frederick Banting and Charles Best Canada Graduate Scholarship awards from the Canadian Institutes of Health Research (CIHR). É Lévesque is a recipient of a ClHR clinician scientist salary award and holds a Prostate Cancer Canada (PCC) Rising Star Award (RS-2013-55). C Guillemette holds the Canada Research Chair in Pharmacogenomics, Tier I.

\section{Author contribution statement}

É Lévesque and C Guillemette conceived the study concept, and designed and supervised the study. L Lacombe and $Y$ Fradet were responsible for patient recruitment and clinical data. I Laverdière, C Flageole, É AudetWalsh, É Lévesque, and C Guillemette were involved in acquisition of data and statistical analysis. P Caron performed the experiments. I Laverdière, C Flageole, É Audet-Walsh, É Lévesque, and C Guillemette wrote the manuscript. All authors contributed to the critical revision of the manuscript for important intellectual content. É Lévesque, C Guillemette, $\mathrm{Y}$ Fradet, and L Lacombe obtained funding for this work.

\section{Acknowledgements}

The authors wish to thank Lyne Villeneuve for technical support. They thank the employees of the genomics and the statistical service platforms of the Centre Hospitalier Universitaire de Québec, particularly Sylvie Desjardins and Sun Makosso-Kallyth respectively.

\section{References}

Audet-Walsh E, Bellemare J, Nadeau G, Lacombe L, Fradet Y, Fradet V, Huang SP, Bao BY, Douville P, Girard H et al. 2011 SRD5A polymorphisms and biochemical failure after radical prostatectomy. European Urology 60 1226-1234. (doi:10.1016/j.eururo.2011.06.020)

Audet-Walsh E, Bellemare J, Lacombe L, Fradet Y, Fradet V, Douville P, Guillemette C \& Levesque E 2012 The impact of germline genetic variations in hydroxysteroid (17- $\beta$ ) dehydrogenases on prostate cancer outcomes after prostatectomy. European Urology 62 88-96. (doi:10.1016/j.eururo.2011.12.021)

Bosland MC \& Mahmoud AM 2011 Hormones and prostate carcinogenesis: androgens and estrogens. Journal of Carcinogenesis 10 33. (doi:10.4103/ 1477-3163.90678)

Boyd LK, Mao X \& Lu YJ 2012 The complexity of prostate cancer: genomic alterations and heterogeneity. Nature Reviews. Urology 9 652-664. (doi:10.1038/nrurol.2012.185)

Cavalieri EL \& Rogan EG 2011 Unbalanced metabolism of endogenous estrogens in the etiology and prevention of human cancer. Journal of Steroid Biochemistry and Molecular Biology 125 169-180. (doi:10.1016/ j.jsbmb.2011.03.008)

Court MH, Zhang X, Ding X, Yee KK, Hesse LM \& Finel M 2012 Quantitative distribution of mRNAs encoding the 19 human UDPglucuronosyltransferase enzymes in 26 adult and 3 fetal tissues. Xenobiotica 42 266-277. (doi:10.3109/00498254.2011.618954)

Gall WE, Zawada G, Mojarrabi B, Tephly TR, Green MD, Coffman BL, Mackenzie PI \& Radominska-Pandya A 1999 Differential glucuronidation of bile acids, androgens and estrogens by human UGT1A3 and 2B7. Journal of Steroid Biochemistry and Molecular Biology 70 101-108. (doi:10.1016/S0960-0760(99)00088-6)

Girard H, Butler LM, Villeneuve L, Millikan RC, Sinha R, Sandler RS \& Guillemette C 2008 UGT1A1 and UGT1A9 functional variants, meat intake, and colon cancer, among Caucasians and African-Americans. Mutation Research 644 56-63. (doi:10.1016/j.mrfmmm.2008.07.002)

Gu Z, Wang G \& Chen W 2014 Estrogen receptor $\alpha$ gene polymorphisms and risk of prostate cancer: a meta-analysis involving 18 studies. Tumour Biology 35 5921-5930. (doi:10.1007/s13277-014-1785-4)

Hu WY, Shi GB, Hu DP, Nelles JL \& Prins GS 2012 Actions of estrogens and endocrine disrupting chemicals on human prostate stem/progenitor cells and prostate cancer risk. Molecular and Cellular Endocrinology 354 63-73. (doi:10.1016/j.mce.2011.08.032)

Kanda S, Tsuchiya N, Narita S, Inoue T, Huang M, Chiba S, Akihama S, Saito M, Numakura K, Tsuruta H et al. 2015 Effects of functional genetic polymorphisms in the CYP19A1 gene on prostate cancer risk and survival. International Journal of Cancer 136 74-82. (doi:10.1002/ ijc.28952)

Kosti O, Xu X, Veenstra TD, Hsing AW, Chu LW, Goldman L, Bebu I, Collins S, Dritschilo A, Lynch JH et al. 2011 Urinary estrogen metabolites and prostate cancer risk: a pilot study. Prostate 71 507-516. (doi:10.1002/pros.21262)

Lepine J, Bernard O, Plante M, Tetu B, Pelletier G, Labrie F, Belanger A \& Guillemette C 2004 Specificity and regioselectivity of the conjugation of estradiol, estrone, and their catecholestrogen and methoxyestrogen metabolites by human uridine diphospho-glucuronosyltransferases expressed in endometrium. Journal of Clinical Endocrinology and Metabolism 89 5222-5232. (doi:10.1210/jc.2004-0331)

Levesque E, Huang SP, Audet-Walsh E, Lacombe L, Bao BY, Fradet Y, Laverdiere I, Rouleau M, Huang CY, Yu CC et al. 2013 Molecular markers in key steroidogenic pathways, circulating steroid levels, and prostate cancer progression. Clinical Cancer Research 19 699-709. (doi:10.1158/1078-0432.CCR-12-2812)

Levesque E, Laverdiere I, Audet-Walsh E, Caron P, Rouleau M, Fradet Y, Lacombe L \& Guillemette C 2014a Steroidogenic germline polymorphism predictors of prostate cancer progression in the estradiol pathway. Clinical Cancer Research 20 2971-2983. (doi:10.1158/10780432.CCR-13-2567)

Levesque E, Laverdiere I, Lacombe L, Caron P, Rouleau M, Turcotte V, Tetu B, Fradet Y \& Guillemette C $2014 b$ Importance of $5 \alpha$-reductase gene polymorphisms on circulating and intraprostatic androgens in prostate cancer. Clinical Cancer Research 20 576-584. (doi:10.1158/1078-0432. CCR-13-1100)

Menard V, Girard H, Harvey M, Perusse L \& Guillemette C 2009 Analysis of inherited genetic variations at the UGT1 locus in the French-Canadian population. Human Mutation 30 677-687. (doi:10.1002/humu.20946) 
Mitsiades N, Sung CC, Schultz N, Danila DC, He B, Eedunuri VK, Fleisher M, Sander C, Sawyers CL \& Scher HI 2012 Distinct patterns of dysregulated expression of enzymes involved in androgen synthesis and metabolism in metastatic prostate cancer tumors. Cancer Research 72 6142-6152. (doi:10.1158/0008-5472.CAN-12-1335)

Mohler JL, Armstrong AJ, Bahnson RR, Boston B, Busby JE, D'Amico AV, Eastham JA, Enke CA, Farrington T, Higano CS et al. 2012 Prostate cancer, version 3.2012: featured updates to the NCCN guidelines. Journal of the National Comprehensive Cancer Network 10 1081-1087.

Montgomery RB, Mostaghel EA, Vessella R, Hess DL, Kalhorn TF, Higano CS, True LD \& Nelson PS 2008 Maintenance of intratumoral androgens in metastatic prostate cancer: a mechanism for castration-resistant tumor growth. Cancer Research 68 4447-4454. (doi:10.1158/0008-5472. CAN-08-0249)

Murai T, Samata N, Iwabuchi H \& Ikeda T 2006 Human UDPglucuronosyltransferase, UGT1A8, glucuronidates dihydrotestosterone to a monoglucuronide and further to a structurally novel diglucuronide. Drug Metabolism and Disposition 34 1102-1108. (doi:10.1124/ dmd.106.009621)

Nadeau G, Bellemare J, Audet-Walsh E, Flageole C, Huang SP, Bao BY, Douville P, Caron P, Fradet Y, Lacombe L et al. 2011 Deletions of the androgen-metabolizing $U G T 2 B$ genes have an effect on circulating steroid levels and biochemical recurrence after radical prostatectomy in localized prostate cancer. Journal of Clinical Endocrinology and Metabolism 96 E1550-E1557. (doi:10.1210/jc.2011-1049)

Nelles JL, Hu WY \& Prins GS 2011 Estrogen action and prostate cancer. Expert Review of Endocrinology \& Metabolism 6 437-451. (doi:10.1586/ eem.11.20)

Reese AC, Pierorazio PM, Han M \& Partin AW 2012 Contemporary evaluation of the National Comprehensive Cancer Network prostate cancer risk classification system. Urology 80 1075-1079. (doi:10.1016/ j.urology.2012.07.040)

Ryan CJ, Smith MR, de Bono JS, Molina A, Logothetis CJ, de Souza P, Fizazi K, Mainwaring P, Piulats JM, Ng S et al. 2013 Abiraterone in metastatic prostate cancer without previous chemotherapy. New England Journal of Medicine 368 138-148. (doi:10.1056/ NEJMoa1209096)

Sneitz N, Vahermo M, Mosorin J, Laakkonen L, Poirier D \& Finel M 2013 Regiospecificity and stereospecificity of human UDP-glucuronosyltransferases in the glucuronidation of estriol, 16-epiestriol, 17-epiestriol, and 13-epiestradiol. Drug Metabolism and Disposition 41 582-591. (doi:10.1124/dmd.112.049072)

Starlard-Davenport A, Xiong Y, Bratton S, Gallus-Zawada A, Finel M \& Radominska-Pandya A 2007 Phenylalanine ${ }^{90}$ and Phenylalanine ${ }^{93}$ are crucial amino acids within the estrogen binding site of the human UDP-glucuronosyltransferase 1A10. Steroids 72 85-94. (doi:10.1016/ j.steroids.2006.11.016)

Stephens M \& Donnelly P 2003 A comparison of bayesian methods for haplotype reconstruction from population genotype data. American Journal of Human Genetics 73 1162-1169. (doi:10.1086/379378)

Stephens M, Smith NJ \& Donnelly P 2001 A new statistical method for haplotype reconstruction from population data. American Journal of Human Genetics 68 978-989. (doi:10.1086/319501)

Tan J, Sharief Y, Hamil KG, Gregory CW, Zang DY, Sar M, Gumerlock PH, deVere White RW, Pretlow TG, Harris SE et al. 1997 Dehydroepiandrosterone activates mutant androgen receptors expressed in the androgen-dependent human prostate cancer xenograft CWR22 and LNCaP cells. Molecular Endocrinology 11 450-459. (doi:10.1210/mend. 11.4.9906)

Tang L, Yao S, Till C, Goodman PJ, Tangen CM, Wu Y, Kristal AR, Platz EA, Neuhouser ML, Stanczyk FZ et al. 2011 Repeat polymorphisms in estrogen metabolism genes and prostate cancer risk: results from the Prostate Cancer Prevention Trial. Carcinogenesis 32 1500-1506. (doi:10.1093/carcin/bgr139)

Varambally S, Yu J, Laxman B, Rhodes DR, Mehra R, Tomlins SA, Shah RB, Chandran U, Monzon FA, Becich MJ et al. 2005 Integrative genomic and proteomic analysis of prostate cancer reveals signatures of metastatic progression. Cancer Cell 8 393-406. (doi:10.1016/j.ccr.2005. 10.001)

Williamson SC, Mitter R, Hepburn AC, Wilson L, Mantilla A, Leung HY, Robson CN \& Heer R 2013 Characterisations of human prostate stem cells reveal deficiency in class I UGT enzymes as a novel mechanism for castration-resistant prostate cancer. British Journal of Cancer 109 950-956. (doi:10.1038/bjc.2013.399)

Wyatt AW, Mo F, Wang Y \& Collins CC 2013 The diverse heterogeneity of molecular alterations in prostate cancer identified through next-generation sequencing. Asian Journal of Andrology 15 301-308. (doi:10.1038/aja.2013.13)

Xu X, Keefer LK, Ziegler RG \& Veenstra TD 2007 A liquid chromatographymass spectrometry method for the quantitative analysis of urinary endogenous estrogen metabolites. Nature Protocols 2 1350-1355. (doi:10.1038/nprot.2007.176)

Zhou J, Tracy TS \& Remmel RP 2010 Glucuronidation of dihydrotestosterone and trans-androsterone by recombinant UDP-glucuronosyltransferase (UGT) 1A4: evidence for multiple UGT1A4 aglycone binding sites. Drug Metabolism and Disposition 38 431-440. (doi:10.1124/dmd.109.028712)

Received in final form 27 November 2014

Accepted 1 December 2014

Made available online as an Accepted Preprint

1 December 2014 http://erc.endocrinology-journals.org DOI: 10.1530/ERC-14-0423
(C) 2015 Society for Endocrinology Printed in Great Britain
Published by Bioscientifica Ltd. 